\title{
Development of a multicompartmental model of the kinetics of quartz dust in the pulmonary region of the lung during chronic inhalation exposure of rats
}

\author{
B A Katsnelson, L K Konysheva, L I Privalova, K I Morosova
}

\begin{abstract}
A multicompartmental model for the kinetics of dust retention in the pulmonary region of the lung and in the tracheobronchial lymph nodes has been developed. The model reflects the following ideas concerning the basic features of this process: (1) penetration into the pulmonary interstitium and translocation to the lymph nodes are possible for nonphagocytised particles only; (2) these processes depend on the degree of damage to macrophages by dust and on the extent of compensatory enhancement in the recruitment of neutrophils taking part in the clearance of the pulmonary region free surface; (3) a certain proportion of initially penetrating particles is continuously returned to this surface together with recruited pulmonary macrophages. The model ensures a satisfactory approximation to experimental data on the retention of quartz in the lung and lymph nodes during long term inhalation exposure, and simulation of the kinetic effects associated with both the damage to and the protection of the macrophagal clearance mechanism.
\end{abstract}

Various mathematical models for the kinetics of deposition and retention of dust in the lung are usually proposed as a basis for predicting "the accumulation of lung burden during prolonged chronic exposure relevant to the exposure of workers to mineral dusts in a wide range of industries" ' 1 and, thus, as an instrument for "exposure assessment for occupational epidemiology". ${ }^{3}$

Another less direct aspect of modelling associated with the same final objective exists, however. Any medical predictions based on the analysis of limited

Medical Research Centre for Occupational Safety and Health, Ekatezinburgh, 620014, USSR

B A Katsnelson, L K Konysheva, L I Privalova, K I Morosova experimental data can be reliable only if the ideas on the physiological and pathogenetic mechanisms of a process under consideration are sound. It is, therefore, necessary first of all to make sure that these ideas have no intrinsic contradictions. This is when mathematical modelling of supposed mechanisms can help by permitting characteristic responses of a real process to corresponding exposure to be reproduced with satisfactory approximation by varying the parameters of a model. Meanwhile, in order to be solved, the problems mentioned ${ }^{1-3}$ do not need any analysis of such mechanisms or their mathematical description with a model.

Modelling the kinetics of the deposition and retention of particles in the "deep lung" (meaning the region that was called "pulmonary" by the ICRP Task Group on Lung Dynamics ${ }^{4}$ and many other authors) Vincent et al considered two mutually independent compartments of "medium" and "slow" clearance; the third compartment (of "fast" clearance) corresponded to the tracheobronchial region. The model, however, does not account for the physiological mechanisms that determine the differences in the rate of clearance of the compartments, and its structure is not claimed to describe actual relations between them.

Most investigators (for example ${ }^{5}$ ) identify the rapidly removed part of dust deposited in the pulmonary region as that which is removed directly from the free surface of the acinus towards the mucociliary escalator, and the slowly removed part as that which has already penetrated into the "fixed tissues" (into the lung interstitium). Thus in both the route of deposition and the route of elimination, real compartments of dust in the "deep lung" are connected in series rather than in parallel as in the model. ${ }^{1}$

One of the variants of this model assumes a sudden and complete failure of the medium clearance compartment and, therefore, transfer of all material into the slow clearance compartment. In another variant of the same model, both compartments have parallel exits not only to the mucociliary escalator but also to some intralung kinetic trap that has no exit at all. Like the basic model, ${ }^{1}$ both of these variants ensure a 


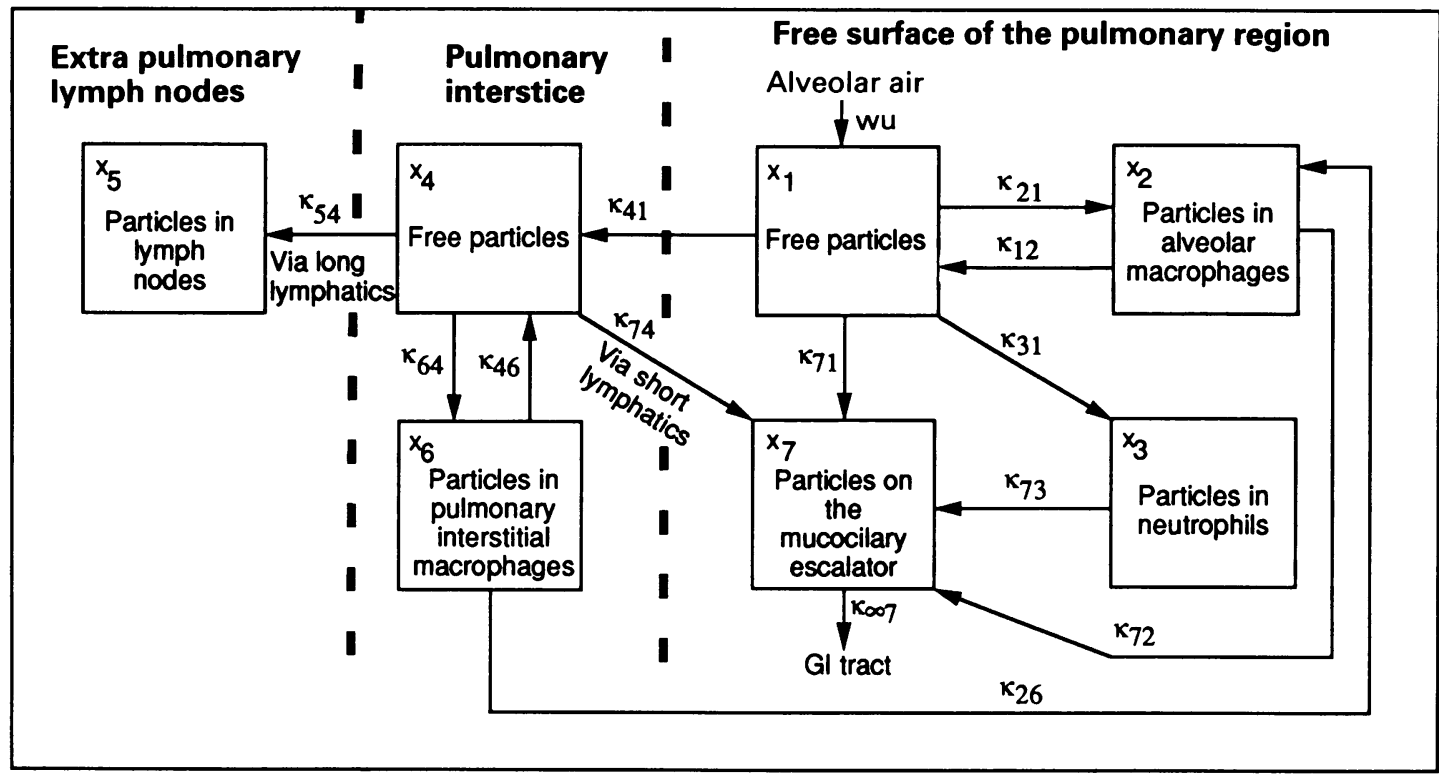

Figure 1 Structure of the multcompartmental model for the kinetics of retention and elimination of dust deposited in the pulmonary region of the lung.

satisfactory approximation to some of the experimental data although they are not based on any mathematical description of the real mechanisms of clearance and retention. As well as the ICRP model, ${ }^{4}$ the model suggested by the Scottish group of authors" may be related to the class of "models of data", rather than to the class of "models of systems" by definition. ${ }^{6}$

Smith, ${ }^{23}$ although pursuing a similar aim in modelling, approached it rather from the position of systems analysis. The structure of this model was originally determined by definite morphofunctional units of the pulmonary region and by the author's ideas on those physiological processes that control the transport of particles deposited in them. Some of these ideas are purely speculative, whereas others seem to be obviously erroneous. As will be shown later, the model cannot simulate some of the essential phenomena observed experimentally. Therefore we have made it our aim to develop further the multicompartmental model of the kinetics of dust in the pulmonary region of the lung based on the approach of constructing it as a "model of systems."

\section{Structure of the model}

Our ideas on the functional mechanisms and routes of clearance of particles from the pulmonary region based on an analysis of published papers and our own experimental data have led us to assume the structure of the multicompartmental model that is shown in fig 1 .

The central compartment $X_{1}$ represents the pool of free particles on a free surface of this region. The only entry to the model is through this compartment and corresponds to the primary deposition of particles in the pulmonary region from the alveolar air. It is described as wu, where $w$ is a constant rate of deposition and $\mathbf{u}=\mathbf{u}(\mathrm{t})$ is the control function, which assumes the value of 1 during actual exposure and the value of 0 during those interruptions that are characteristic of both occupational exposure and, frequently, experimental chronic exposure. The problem can be simplified for many conditions, as deposition throughout exposure may be assumed to proceed continuously, but at an averaged value of $\bar{w}$.

The arrows between the compartments of the model show the direction of dust transport, and the constants $k_{\mathrm{ji}}$ near these arrows represent the proportions of dust transferred from the compartment $\mathrm{X}_{\mathrm{i}}$ to the compartment $\mathrm{X}_{\mathrm{i}}$ per unit time (the transfer rate constants).

Particles ingested by alveolar macrophages (AMs) or neutrophils (NLs) on the free surface of the pulmonary area are described by the compartments $\mathrm{X}_{2}$ and $\mathrm{X}_{3}$ respectively. Note that allowing for the essential role of phagocytosis by neutrophils as a further mechanism of pulmonary clearance, proved in our studies, ${ }^{78}$ is the first basic feature of the model proposed. This makes it different from Smith's model. ${ }^{23}$ 
The second difference lies in the method used to describe the cytotoxic action of particles on the kinetics of their transfer from $X_{1}$ to $X_{2}$. The model of Smith $^{23}$ is non-linear: the rate of transfer of particles to the macrophage compartment is inversely dependent on the amount of dust already present in it. Our model, too, assumes the possibility of a decrease in this rate. This is due, however, not to the amount of dust as it is, but to the extent of pathological change in the lung caused by it, as was shown earlier. ${ }^{8}$ The coefficient $k_{21}$ is assumed to be constant both before and after a "breakdown" of the macrophage clearance mechanism (this breakdown being simulated by a single change in the value of this coefficient). At the same time, our model describes the obvious effect of continuous cytotoxic damage to AMs by phagocytosed dust, which was ignored by Smith $^{2} 3$-namely, disintegration of AMs as a result of which phagocytosed particles become free again. In the model shown in fig 1 this corresponds to transfer from the compartment $X_{2}$ to the compartment $X_{1}$ with rate constant $k_{12}$.

A single NL contains on average considerably fewer phagocytosed particles than a single AM and is very rarely seen to be damaged. Therefore, reverse transfer from $X_{3}$ to $X_{1}$ is assumed to be negligible and is not described by the model.

The compartments $X_{1}, X_{2}$, and $X_{3}$ have exits to the compartment $X_{7}$ that corresponds to the mucociliary escalator-that is, outside the pulmonary region and related to the tracheobronchial region (with an exit to the gastrointestinal tract). For all the three flows of dust to the compartment $\mathrm{X}_{7}$ from the compartments describing free or phagocytosed particles on the free surface of the pulmonary region, the model does not distinguish between the transfer of particles-or cells that have ingested them-over this free surface and the transfer of particles through hypothetical "liquid veins".

As in the model of Smith, ${ }^{23}$ penetration into the interstitium is assumed to be possible only for free particles and is described by the transfer from the compartment $\mathrm{X}_{1}$ to the compartment $\mathrm{X}_{4}$ with rate constant $k_{41}$. The further fate of these particles in the model of Smith ${ }^{23}$ is described as either transfer into lymph nodes or encapsulation out of which particles cannot break themselves loose and clearance may proceed only through their gradual dissolution. The first type of transfer is shown in our model too; the flow from the compartment $X_{4}$ to the compartment $X_{5}$ corresponding to the dust in lymph nodes, and the constant $k_{54}$. As regards the socalled encapsulation it is well known that particles can even be transferred from collagenous and hyalinised silicotic lesions with the participation of interstitial lung macrophages. ${ }^{1011}$ On the other hand, long before such lesions are formed, macrophagal phagocytosis of particles that have penetrated into the interstitium is a permanen- tly acting mechanism of their more or less prolonged fixation in this region, whereas the damage to and destruction of interstitial macrophages by particles triggers silicotic pathogenesis. Meanwhile, from the standpoint of kinetic analysis this destruction means that particles break out of the phagocytosed state and become free again. In our model these processes are represented by the two way connection between the compartment $\mathrm{X}_{4}$ and the compartment $\mathrm{X}_{6}$ with corresponding constants $k_{64}$ and $k_{46}$.

Our model assumes a negligibly low solubility of particles and therefore it has no exits determined by the rate of dissolution. For a substance with a sufficiently high solubility to have a pronounced effect on the clearance of the pulmonary region (for example, from particles of colloid silicic acid), ${ }^{12}$ all the compartments of the model have to be provided with corresponding exits. It should be emphasised that even practically insoluble dusts are often seen to reach an equilibrium level of retention during chronic inhalation exposure, ${ }^{813}$ and after exposure has ceased, the pulmonary area in rats may be actively cleared even of the dust that has accumulated in the interstitial tissues (although in other cases the phenomenon of dust sequestration described by model ${ }^{1}$ does exist). It is impossible to describe these experimental data with the help of the model of Smith, ${ }^{23}$ unless an unrealistic rate constant for the transfer of particles from the lung to the lymph nodes is assumed. We think that the absence of the backward flow from the interstitium to the free surface of the respiratory tract is a serious drawback of the model of Smith. ${ }^{23}$

We have analysed two variants of modelling such a backward flow, proceeding from two basically different concepts. The first of the variants was considered in accordance with ideas of Tucker et $a^{14}$ who maintained that the particles that have penetrated into the lung interstitium without the participation of phagocytosis can go further either into the long lung drainage pathways that transport them to the lymph nodes or into the short pathways opening into the terminal bronchiole at the beginning of the mucociliary escalator. Figure 1 shows the last path as a flow from the compartment $X_{4}$ to the compartment $X_{7}$ with rate constant $k_{74}$. The model successfully simulates the gradual levelling off of dust retention in the lung. Nevertheless, it is not suitable for simulating some other important kinetic relations and processes.

Thus it is well known that protection of macrophages against damage by quartz reduces the retention of dust in the lung. ${ }^{15}$ Modelling this protection by reducing the constant $k_{46}$ and increasing the constant $k_{64}$, however, (see later), we increase the amount of dust in the compartment $\mathrm{X}_{6}$ at the expense of its reduction in the compartment $\mathrm{X}_{4}$ and thereby reduce the flow from $\mathrm{X}_{4}$ to $\mathrm{X}_{7}$, which brings about an 
increase in the total retention of dust in the lung. On the other hand, the presence of flow from $X_{4}$ to $X_{7}$, similarly does not allow the model to adequately simulate more substantial retention of more cytotoxic dusts in the lung as compared with less cytotoxic dusts, a well known experimental fact.

Therefore we gave up this variant and considered that in which $k_{74}=0$, but with a flow from the compartment $\mathrm{X}_{6}$ to the compartment $\mathrm{X}_{2}$. Introducing this flow into the model we proceeded from the fact that the pool of interstitial macrophages is one of the main sources for recruiting AMs. ${ }^{516}$ Reproduction of the cells of this pool is stimulated by macrophage breakdown products; the same products enhance their recruitment as AMs on the free surface of alveoli. ${ }^{17}$ On the other hand, the breakdown of AMs increases the probability of particle penetration and, consequently, the probability of their ingestion by interstitial macrophages. Thus the breakdown of AMs creates conditions for both increased transfer of cells from the interstitial pool to the alveolar pool of lung macrophages, and for part of these cells to contain some particles already before this transfer. The recruitment of macrophages becomes a mechanism of returning a proportion of the interstitial particles on to the free surface of the pulmonary region, but already in a phagocytosed state. This reverse transfer proceeds at a rate which, in our model, is characterised by the constant $k_{26}$.

Apparently the same increase in the constant $k_{64}$ and decrease in the constant $k_{46}$ leading to the transfer of dust to the compartment $\mathrm{X}_{6}$ at the expense of the compartment $\mathrm{X}_{4}$ facilitate the elimination of dust from the lung towards the free surface in this model rather than impede it as in the first variant.

Mathematically the equivalent of the model shown in fig 1 is represented by a set of linear differential equations.

\section{Estimating model constants: general considerations}

The basic difficulties that arise during quantitative estimation of the parameters pertaining to a multicompartmental model of the kinetics of dust in the pulmonary region of the lung during chronic inhalation exposure are associated with the fact that direct measurement of the amount of dust in the morphofunctional divisions of this area that are described by corresponding compartments of the model is impossible. Even the extrapulmonary compartment $X_{5}$ can be considered as an analogue of dust deposited in tracheobronchial lymph nodes only under some assumptions. As regards the second quantity available for an experimenter-namely, the amount of dust in the lung parenchyma-it is only at first sight that it corresponds to the sum of the other six compartments of our model. The possibility of describing satisfactorily the kinetics of dust retention in the lung parenchyma during chronic inhalation exposure by means of a single exponential function ${ }^{813}$ shows that this retention relates mainly to the region that may be assumed as isokinetic. In other words, we may assume that the amount of dust found in the lung after a many week exposure depends mainly on those particles that have been retained in "fixed tissues" (the lung interstitium), whereas the contribution to this amount of particles remaining on the free surface of the intralung airways becomes comparable with the error of its determination. Clearly the longer the period of exposure elapsed-that is, the higher the retention of dust in the lung interstitium as compared with its retention on the free surface of the pulmonary region and bronchi (that reaches equilibrium much earlier)-the more valid this assumption becomes.

Moreover, for modelling we usually have the data of such experiments in which rats are killed one day after a final inhalation exposure-that is, enough time exists for substantial clearance of the free surface from deposited particles. Finally, for estimating the role of phagocytosis in the clearance of these particles it was necessary, of course, to perform a bronchoalveolar lavage that also clears the lung from particles that have not penetrated into the interstitium.

Thus sufficient reason exists to assume the experimentally determined lung burden (especially during long term exposure) as roughly equal to the total amount of dust in the compartments $\mathrm{X}_{4}$ and $\mathrm{X}_{6}$, which in our model represent the dust in the lung interstitium.

At the same time temporal changes in the amount of dust during exposure are satisfactorily approximated by the function $\mathrm{y}=\mathrm{a}-\mathrm{be}^{-\mathrm{kt}}$. $^{8}$ Therefore an empirical model for the retention of dust in the pulmonary area constructed on the basis of such data may be definitively identified as a single compartment one. Using $k$ as a constant of the rate of dust elimination from the compartment the rate of deposition of dust in it from the alveolar air (the constant $\bar{w}$ ) can be easily calculated, if for simplifying the problem exposure is assumed to be continuous $(u(t)=1$ during the entire period). Then, proceeding to construct a multicompartmental model of the system the same value for $\bar{w}$ as a constant rate of entry of dust into it can be assumed.

One more experimental estimate can be used to check the adequacy of multicompartment modelling. This is the ratio $[N L] \times C_{n l} /[A M] \times C_{a m}$, where [NL] and [AM] are the neutrophil and alveolar macrophage counts in the bronchoalveolar lavage (BAL), $C_{n 1}$ and $C_{a m}$ are the average numbers of dust particles detected in a single NL or AM by light microscopy of the BAL cell sediment. We think that it is not necessary to explain why this ratio can be 
only a rough estimate of the $\mathrm{X}_{3}$ to $\mathrm{X}_{2}$ dust burden ratio. Nevertheless, these two ratios should be at least of the same order of magnitude, and a large difference between them would be evidence of an erroneous choice of the model constants.

Thus for a given value of $\bar{w}$ a set of constants $k_{j i}$ should ensure such a prediction of the amount of dust in the model compartments for all experimental durations of exposure that would give a sum $\left(X_{4}+X_{6}\right)$ in sufficiently close agreement with the amount of dust determined in the lung tissue, and $X_{5}$ in agreement with the amount of dust in tracheobronchial lymph nodes. Also the $\mathrm{X}_{3} / \mathrm{X}_{2}$ ratio would not differ very much from the ratio [NL] $\times \mathrm{C}_{\mathrm{nl}} /$ [AM] $\times \mathrm{C}_{\mathrm{am}}$. Besides, the model should reproduce the tendency of dust retention in the compartment $\left(\mathrm{X}_{4}+\mathrm{X}_{6}\right)$ to level off into equilibrium as found in experiments on rats sometime between the 20th and 40th weeks of exposure. ${ }^{8}$

Of course, having constructed a seven compartment model for the identificaton of which there are only limited experimental data, satisfactory agreement with these data can be obtained using various sets of constants $\mathrm{k}_{\mathrm{ji}}$. Theoretical considerations and some experimental data suggest certain limitations, which are discussed next.

\section{Estimating model parameters: simulation of experimental data}

For estimating the model parameters we used primarily the results of the experiment described earlier, ${ }^{8}$ in which rats inhaled quartz dust in a whole body type chamber at an average concentration of 87.8 (SE 3.4) $\mathrm{mg} / \mathrm{m}^{3}$ (total dust) five hours a day, five times a week for $10,20,40$, or 48 weeks. The lung dust burden was determined after a bronchoalveolar lavage carried out 24 hours after a final inhalation exposure. The growth of this mass was described by the function $y=21-17 \mathrm{e}^{-0.0783 t}$, where $t$ is the duration of exposure in weeks. The value of $\bar{w}$ calculated by the above method is equal to $1.644 \mathrm{mg}$ week $^{-1}$. The coefficient $k=0.0783$ week $^{-1}$ was maintained constant throughout the entire 48 week period, despite the fact that between the 20th and 40th weeks, there was a permanent "breakdown" of the mechanisms of enhanced AM recruitment as a response to exposure. This constancy was attributed to the observed compensatory increase in NL recruitment. $^{8}$

Thus the problem of modelling involved not only selection of adequate values for the constants of our seven compartment model, but simulation of the breakdown of the macrophage clearance mechanism and its neutrophilic compensation as well. When selecting the basic set of constants to describe the initial state of the clearance and translocation mechanisms we assumed, as already mentioned, certain limitations and preliminary estimates.
Thus we assumed that lung macrophages from both pools (alveolar and interstitial) cannot differ greatly from each other in either the phagocytic activity or the resistance to cytotoxic damage by phagocytosed dust particles. At the same time, an alveolar macrophage functions on the surface where the concentration of deposited inhaled particles is highest and the conditions for the migration of cells towards these particles are most favourable. It can therefore be assumed that the probability of phagocytosis here is higher than in the lung interstitium, and, hence, the probability of such a cytotoxic load is higher on individual macrophages. This would lead to their damage and eventual breakdown. All these considerations make it clear that $k_{21}$ cannot differ too greatly from $k_{64}$; neither can $k_{12}$ from $k_{46}$, but it is essential that the conditions $k_{21}>k_{64}$ and $k_{12}>k_{46}$ be satisfied.

Furthermore, as the contribution of the macrophage clearance mechanism before its breakdown is considerably greater than that of the neutrophilic mechanism, the constant $k_{21}$ in the base model should be assumed to have a value much greater than that of the constant $k_{31}$. Specifically, comparison of the values for $[A M] \times C_{a m}$ and $[N L] \times C_{n l}$ shows that by the first two terms of exposure the total phagocytic activity of all AMs in the BAL was 20 and 10 times as high as the total activity of all NLs. Therefore, we presume that the difference between the constants $k_{21}$ and $\mathrm{k}_{31}$ should be at least 10 -fold.

By contrast, the rate constants for two flows bringing phagocytosed dust into the compartment $\mathrm{X}_{7}$ should, apparently, be sufficiently close. Irrespective of whether the transfer of coniophages from the free surface of alveoli to the zone of the mucociliary escalator occurs over the surface or by "liquid veins," the dominating view in modern science is that this transfer, as well as the corresponding transfer of free particles, is passive. It takes place under the action of physical forces associated with respiratory excursions ("tidal force" or "squeezing out"). This indicates a necessity to assume the constants $k_{72}$ and $k_{73}$ to be, if not equal, then at least of the same order of magnitude. Although the preliminary packaging of dust in a macrophage has been claimed to facilitate its transfer, ${ }^{18}$ the same authors point to the danger of formation of a cell stasis in the mouth of the acinus that can block its self clearance. Rather, a large cell is transported under the action of the mentioned physical forces slower than free particles and smaller cells. For these reasons $\mathrm{k}_{72}$ may be assumed to have a somewhat lower value than $k_{73}$ rather than the opposite.

Once in the zone of action of mucociliary transport, dust is apparently cleared at about the same rate as inhaled particles initially deposited in the tracheobronchial region. In accordance with experimental estimates (for example, ${ }^{19}$ ) for rats this 
process is characterised by $T_{1}$ equal to about $20-24$ hours. Hence, the constant $k_{\infty 7}$ should be close to 5.0 weeks ${ }^{-1}$.

Under all these limitations, we sought such a set of model constants for which the retention of dust predicted by this set would correspond satisfactorily to experimental data (see the previous section). Table 1 (column "before breakdown") presents the set we chose as giving a satisfactory approximation to experimental data. All of the above mentioned limitations are met. By the end of the first half of the experiment, (at the end of 20 weeks) the model with these constants gave the following predictions of dust retention: $15.0 \mathrm{mg}$ in the compartments $\left(\mathbf{X}_{\mathbf{4}}+\mathbf{X}_{6}\right)$ and $0.86 \mathrm{mg}$ in the compartment $X_{5}$. The amount of dust in the lung tissues determined by that time was equal to 16.9 (SE 2.2) $\mathrm{mg}$ with $95 \%$ confidence intervals $(95 \% \mathrm{CIs})$ of $12.1-21.7 \mathrm{mg}$; that in the tracheobronchial lymph nodes was $1.08 \mathrm{mg}$.

Predictions for the 10 week period showed worse agreement with experimental data: if the model predicts $7.8 \mathrm{mg}$ for the compartment $\left(\mathrm{X}_{4}+\mathrm{X}_{6}\right)$, the experimental result is 13.7 (SE 1.7) $\mathrm{mg}$, even the lower CI being higher than the model prediction. It should be noted, however, that the model assumes the lungs to be initially completely free from dust, whereas dust was found even in lungs of control rats in the same experiment. ${ }^{8}$

The next step in modelling consisted of simulating the breakdown of the macrophage clearing mechanism mentioned earlier. We thought is necessary to assume two important simplifications of the problem. Firstly, the process, which most probably proceeds gradually, was simulated by a single change in model constants immediately after the 20 th week. Secondly, although the most prominent manifestation of a breakdown was a reduction in AM recruitment, ${ }^{8}$ its effect on the kinetics of clearance could be estimated experimentally by the decrease in the total number of particles phagocytosed by all free AMs. Basically, this decrease is a sum of two effects: (a) a reduction in the number of AMs leading to slower phagocytosis of particles on the free surface of alveoli, and (b) a decrease in the number of particles brought there already in a phagocytosed state from the interstitium. In other words, one should have simulated the phenomenon of breakdown by decreasing the constants $k_{21}$ and $k_{26}$. There is every reason to think, however, that the corresponding flows are not comparable, and that the flow from $\mathrm{X}_{1}$ gives a dominant contribution of $\mathrm{X}_{2}$. Therefore, we considered it possible to limit ourselves to the reduction of the constant $k_{21}$. After 40 weeks of exposure the index [AM] $\times \mathrm{C}_{2 \mathrm{~m}}$ in the BAL was 1.7 times lower than that after 20 weeks. As more accurate estimates were not available, we thought it sufficient to reduce $k_{21}$ also by a factor of 1.7 for simulating the breakdown under consideration.

As was already mentioned, such a breakdown was claimed to be entirely offset by an increase in the number of NLs and thus enhanced phagocytosis by neutrophils. ${ }^{8}$ Consequently, it was necessary to find such an increase in the constant $k_{31}$, which would give predictions for the amount of dust in $\mathrm{X}_{4}, \mathrm{X}_{5}$, and $\mathrm{X}_{6}$ by the 40th week and 48th week of exposure that would agree with experimental results, all the other parameters of the basic model being unchanged.

Such agreement was obtained by increasing $k_{31}$ by a factor of 15 (see table 1; the column "after breakdown"), the model predicting $20.47 \mathrm{mg}$ of dust in $\left(X_{4}+X_{6}\right)$ after 40 weeks and $21.76 \mathrm{mg}$ after 48 weeks. These values are close to the experimental data on the amount of dust in the lung tissue (19.4 (SE 1.7) $\mathrm{mg}$ and 20.8 (SE 3.4) $\mathrm{mg}$ respectively). The predictions for the compartment $\mathrm{X}_{5}$ for the same period of exposure were $2.42 \mathrm{mg}$ and $3.1 \mathrm{mg}$; an experimental result is available for 48 weeks only and is close to the prediction- $-3.5 \mathrm{mg}$.

Table 1 The constants of the seven compartment model for the kinetics of quartz dust in the pulmonary region of the lung and lymph nodes of rats

\begin{tabular}{|c|c|c|c|c|c|}
\hline \multirow[b]{3}{*}{$k_{n}\left(w_{e e k^{-1}}\right)$} & \multicolumn{2}{|c|}{ By the results of experiment ${ }^{8}$} & \multicolumn{3}{|c|}{ By the results of experiment ${ }^{\prime s}$} \\
\hline & \multirow{2}{*}{ Before "breakdown" } & \multirow[b]{2}{*}{ After "breakdown" } & \multirow[b]{2}{*}{ “Adapted" model } & \multicolumn{2}{|c|}{ Simulation of glutamate effects } \\
\hline & & & & $0-12$ weeks & After 12 weeks \\
\hline $\begin{array}{l}\mathbf{k}_{12} \\
\mathbf{k}_{21} \\
\mathbf{k}_{26} \\
\mathbf{k}_{31} \\
\mathbf{k}_{41} \\
\mathbf{k}_{46} \\
\mathbf{k}_{54} \\
\mathbf{k}_{64} \\
\mathbf{k}_{71} \\
\mathbf{k}_{72} \\
\mathbf{k}_{73} \\
\mathbf{k}_{74} \\
\mathbf{k}_{17}\end{array}$ & $\begin{array}{l}0.05 \\
0.25 \\
0.06 \\
0.02 \\
0.50 \\
0.03 \\
0.01 \\
0.15 \\
0.10 \\
0.05 \\
0.10 \\
0.00 \\
5.00\end{array}$ & $\begin{array}{l}0.05 \\
0.15 \\
0.06 \\
0.30 \\
0.50 \\
0.03 \\
0.01 \\
0.15 \\
0.10 \\
0.05 \\
0.10 \\
0.00 \\
5.00\end{array}$ & $\begin{array}{l}0.05 \\
0.25 \\
0.06 \\
0.04 \\
0.60 \\
0.03 \\
0.025 \\
0 \cdot 15 \\
0.01 \\
0.05 \\
0 \cdot 10 \\
0.00 \\
5.00\end{array}$ & $\begin{array}{l}0.005 \\
0.75 \\
0.06 \\
0.027 \\
0 \cdot 60 \\
0.003 \\
0.025 \\
0.45 \\
0.01 \\
0.05 \\
0 \cdot 10 \\
0.00 \\
5.00\end{array}$ & $\begin{array}{l}0.003 \\
1.125 \\
0.06 \\
0.02 \\
0.60 \\
0.002 \\
0.025 \\
0.675 \\
0.01 \\
0.05 \\
0 \cdot 10 \\
0.00 \\
5.00\end{array}$ \\
\hline
\end{tabular}




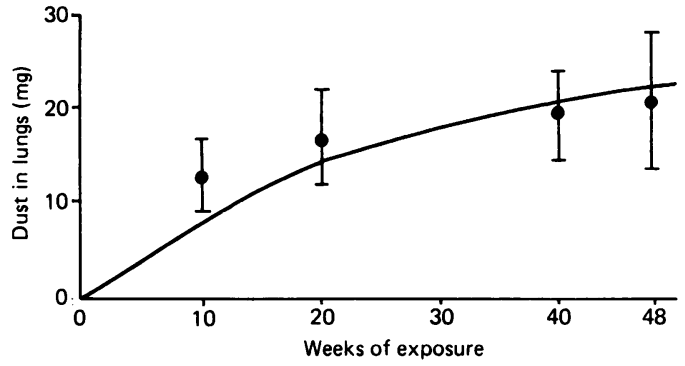

Figure 2 Retention of dust in the lung after 10, 20, 40, and 48 weeks of exposure to quartz by experimental data (mean with $95 \% \mathrm{CI}$ ) and the curve showing accumulation of dust by model predictions.

The index [NL] $\times \mathrm{C}_{\mathrm{nl}}$ in the BAL for the second half of the experiment was higher than for the first half by a factor of up to 14.3. This estimate shows that a 15-fold increase in the corresponding constant required by the model is not unrealistic.

If we simulate a cessation of exposure after 40 weeks (assuming $\overline{\mathbf{w}}=0$ ), all the other parameters of the model with a breakdown and compensation being unchanged, the model predicts $16.6 \mathrm{mg}$ of dust in the compartment $\left(\mathrm{X}_{4}+\mathrm{X}_{6}\right)$ by the 48 th week. In the experiment, the rats for which exposure was stopped after the 40th week and which were killed eight weeks later had 13.8 (SE 1.4) $\mathrm{mg}$ of dust in lung tissue with $95 \% \mathrm{CI}$ of $10.7-16.9 \mathrm{mg}$. Thus this result also shows the adequacy of the model.

The experimental estimate of the ratio [NL] $\times$ $\mathrm{C}_{\mathrm{n} /} /[\mathrm{AM}] \times \mathrm{C}_{\mathrm{am}}$ during the first two periods of exposure was 0.081 on average, and during the last two periods it was $0 \cdot 710$. For the dust burden ratio $\mathrm{X}_{3} / \mathrm{X}_{2}$, the model predicts 0.068 by the 10 th week, 0.053 by the 20 th week, 0.599 by the 40 th week, and 0.563 by the 48 th week.

Figure 2 shows that the model also satisfies the requirement of gradual levelling off for the amount of dust in $\left(\mathbf{X}_{4}+\mathbf{X}_{6}\right)$ within periods of time comparable with the experimental data on such levelling off in rats.

Of course, the numerical parameters of the model that ensure a satisfactory approximation to particular experimental data are not universal. The unquestionable biological variability of the mechanisms involved in the kinetics of elimination, retention, and translocation of particles deposited in the pulmonary region, and the effects of chronic exposure conditions on these mechanisms make the adjustment of a specific set of constants for each experiment inevitable. Only the above described limitations on such adjustment are more or less general. The very possibility of describing the results of another experiment using a model of the same structure is a serious argument in favour of this structure, and those concepts included in its basis. As we stated in the introduction, this is the main objective of our modelling. Nevertheless, we have had an opportunity to also make sure that such description requires only minimal adaptation of model parameters estimated by the data from the original experiment.

Such adaptation was applied to the results of another experiment also described in this journal. ${ }^{15}$ In this case, rats inhaled the same quartz dust, but at a lower concentration $\left(71.3(\mathrm{SE} 1.9) \mathrm{mg} / \mathrm{m}^{3}\right)$, which led us primarily to change the rate of entry of dust into $X_{1}$ assuming it to be equal to $1.3608 \mathrm{mg} \mathrm{week}^{-1}$. When comparing the cell composition of the BAL in both experiments described ${ }^{815}$ we noticed that the number of AMs for corresponding periods of exposure did not differ greatly, but the number of NLs in one experiment ${ }^{15}$ was twice as great as in the other. ${ }^{8}$ This led us to assume twice the value for the constant $k_{31}$. Further adjustment of constants showed that a satisfactory approximation to the data of this experiment also required a higher probability of nonphagocytosed particles penetrating into the interstitium (with a corresponding decrease in the alternative probability of their being transferred to the zone of the mucociliary transport), and a higher probability of their subsequent transfer to lymph nodes. These mutually non-contradicting assumptions were reflected by a corresponding change in the constants $k_{41}, k_{71}$, and $k_{54}$ (see table 1 , column "adapted model").

As table 2 shows, model predictions were in satisfactory agreement with experimental data for both terms of the investigation, which in this experiment corresponded to 12 and 23 weeks of exposure.

In the same experiment, ${ }^{15}$ the rats that were placed into the same dust chamber but given a solution of sodium glutamate instead of drinking water, had a strongly reduced retention of dust in the lungs and especially in lymph nodes owing to a considerably increased resistance of macrophages to the cytotoxic action of particles engulfed by them. A direct indication of this increased resistance was a decrease in the percentage of clearly degenerate AMs in the cell population of the BAL, and indirect evidence was provided by a reduced ratio NL/AM, which is an adequate measure of the cytotoxic action of dust. ${ }^{\text {? }}$

The most obvious kinetic consequences of this cytoprotective action of glutamate are as follows:

(a) A resistant macrophage retains its functional activity and integrity at a considerably greater number of engulfed cytotoxic particles. Hence many more cells still retain phagocytic activity under the action of the cytoprotector (despite the dose of cytotoxic particles already engulfed) per finite number of cells, than in the absence of this action. Considering the cell population as a whole the same effect can be 
Table 2 Simulaton of the kinetic effects of glutamate by an adapted seven compartment model

\begin{tabular}{|c|c|c|c|c|c|c|c|}
\hline \multirow[b]{2}{*}{ Drink } & \multirow{2}{*}{$\begin{array}{l}\text { Duration of } \\
\text { exposure to } \\
\text { dust (weeks) }\end{array}$} & \multicolumn{3}{|l|}{ Experiment } & \multicolumn{3}{|l|}{ Model } \\
\hline & & $\left\{\begin{array}{l}N L \times C_{n l} \\
A M \times C_{a m}\end{array}\right.$ & $\begin{array}{l}\text { Dust in lungs } \\
(m g(S E))\end{array}$ & $\begin{array}{l}\text { Dust in lymph } \\
\text { nodes }^{\star}(\mathrm{mg})\end{array}$ & $X_{3} / X_{2}$ & $\left(X_{4}+X_{6}\right)(m g)$ & $X_{5}(m g)$ \\
\hline & 12 & $0 \cdot 12$ & $\begin{array}{c}7 \cdot 0(0 \cdot 9) \\
5 \cdot 1-8 \cdot 9 \dagger\end{array}$ & 1.06 & $0 \cdot 127$ & $8 \cdot 60$ & $0 \cdot 84$ \\
\hline Water & 23 & $0 \cdot 16$ & \multirow{3}{*}{$\begin{array}{c}16 \cdot 0(1 \cdot 8) \\
12 \cdot 2-19 \cdot 8 \dagger \\
5 \cdot 6(0 \cdot 4) \\
4 \cdot 8-6 \cdot 4 \dagger \\
5 \cdot 8(0.5) \\
4 \cdot 8-6.9 \dagger\end{array}$} & $2 \cdot 22$ & 0.094 & $14 \cdot 80$ & 2.51 \\
\hline $\begin{array}{l}1.5 \% \text { solution } \\
\text { of sodium }\end{array}$ & 12 & 0.06 & & $0 \cdot 14$ & 0.020 & $5 \cdot 20$ & $0 \cdot 29$ \\
\hline glutamate & 23 & 0.09 & & 0.51 & 0.012 & 6.78 & 0.50 \\
\hline
\end{tabular}

^dust content was determined in pooled lymph node tissue of all rats of a group.

$+95 \%$ CI.

expressed as an increase in its phagocytic activity. In our model this effect can be simulated by increasing the constants $k_{21}$ and $k_{64}$.

(b) Another aspect of the same cytoprotective effect is, of course, a lower probability that particles taken up by macrophages will be released again, which may be simulated by decreasing the constants $k_{12}$ and $k_{46}$.

(c) Reduction in the compensatory recruitment of NLs-that is, a considerable decrease in the total number of NLs on the free surface of the pulmonary area under the action of glutamate- reduces the probability that a free particle on it will be phagocytosed by these cells (in the model, transferred from $\mathrm{X}_{1}$ to $\mathrm{X}_{3}$ ). This consideration requires a reduction in the constant $\mathrm{k}_{31}$.

Taking as a guideline the comparative estimate of the number of NLs in the BAL of rats that inhaled quartz dust against the background of drinking glutamate solution or water, this constant could be reduced by a factor of 1.5 to 2 . For preliminary estimates of adequate changes in other constants no direct experimental data exists. Therefore the task of modelling involved finding such changes in the above constants to which the model would respond by a substantial decrease in dust retention in the compartments $\left(\mathbf{X}_{4}+\mathbf{X}_{6}\right)$ and an even more pronounced decrease in the compartment $\mathrm{X}_{5}$, and by a decrease in the ratio $\mathrm{X}_{3} / \mathrm{X}_{2}$-that is, would simulate the basic kinetic effects of glutamate. We considered it reasonable to assume that changes in the constants $k_{21}$ and $k_{12}$ are equal to changes in the constants $k_{64}$ and $k_{46}$ respectively.

At the same time, experimental data show that with an increased period of exposure to dust the favourable kinetic effect of glutamate builds up: eventually this leads to an ever growing difference in dust retention in the lung between the groups that were and were not given glutamate (see table 2). Apparently, this can be attributed to the build up in the cytoprotective effect of glutamate. Indeed, the results published earlier ${ }^{15}$ show that after 12 weeks of exposure the percentage of degenerate AMs in the
BAL of rats that inhaled quartz and were given glutamate was lower by a factor of 2.6 and the ratio NL/AM by a factor of 1.4 than in rats that were not. After 23 weeks, these ratios were 4.5 and 2.0 respectively. If we take these values as relative estimates of the antiquartz cytoprotective effect, we can come to the conclusion that this was enhanced during the second half of the period by a factor of 1.73 judging by the first index and by about 1.43 judging by the second. Although this enhancement most likely proceeded gradually, its simulation proved to be possible by a single change in the constants.

Thus the simulation of the kinetic effect of glutamate was performed in two steps (see table 1). Firstly, we found, in accordance with the basic premises, such changes in the constants $k_{12}, k_{21}, k_{46}$, $k_{64}$, and $k_{31}$ that ensured satisfactory approximation to the experimental data relating to the 12 th week. Then the constants $k_{21}$ and $k_{64}$ were further increased, whereas the constants $k_{12}, k_{46}$, and $k_{31}$ were further reduced by a factor of about $1 \cdot 5$. Table 2 shows that this approach gives satisfactory results on the whole. The model predictions for the compartments $\left(\mathbf{X}_{4}+\mathbf{X}_{6}\right)$ for the first period were practically equal to the average mass of dust in the lung, and for the second period it was within the $95 \%$ CIs of this value. Moreover, the model simulated the above mentioned build up in the favourable effect of glutamate on dust retention in the lung: if during the first period of exposure the amount of dust in $\left(\mathrm{X}_{4}+\mathrm{X}_{6}\right)$ was lower than the prediction of the adapted model without simulating the cytoprotective action only by a factor of $1 \cdot 65$, by the second period it was lower by a factor of $2 \cdot 18$.

The prediction for the compartment $\mathrm{X}_{5}$ was almost equal to the amount of dust in lymph nodes during the second period, although it was less accurate during the first. In compliance with the main objective of the modelling, however, the simulation of the principal effect is of the greatest importance. This effect consists of the fact that any cytoprotector reduces cytotoxic damage by dust not only to alveolar 
but to interstitial macrophages as well, thereby reducing the penetration of particles into lymph nodes to an even greater extent than their penetration into the lung interstitium. Indeed, table 2 shows that the mass of dust in the lung decreased under the action of glutamate-for example, by the second period-by a factor of 2.76 compared with its retention in rats that were fed water, whereas in lymph nodes it changed by a factor of $4 \cdot 35$. The model gave a decrease by a factor of $2 \cdot 18$ and 5.02 respectively. Both experimental and predicted estimates show the predominant effect of glutamate on the retention of dust in lymph nodes during the first period as well.

As with all the previous solutions, the predictions of the $\mathrm{X}_{3} / \mathrm{X}_{2}$ ratio were in agreement with the experimental estimates of the numerical ratio $[N L] \times C_{n l} /[A M] \times C_{a m}$ only in the order of magnitude. In this case also, the most important thing is that the model simulated the basic tendency to a shift in this ratio as caused by glutamate, namely, a pronounced decrease in it.

Previously we proposed the so called "action integral (AI)" as a measure of the chronic harmful influence of pulmonary dust burden. ${ }^{820}$ The AI is a definite integral of the function describing accumulation of any substance in and its elimination from the organism (or any of its parts presented as a compartment in a toxicokinetic model) computed in the given time boundaries. The program elaborated for estimating the parameters of the model under consideration permits the computation of the AI for all of its compartments. Taking into account, however, that the breakdown of interstitial pulmonary macrophages triggers the release of the silicotic fibrogenic factor and induces other pathological consequences characteristic of silicosis, we considered the AI for the $\mathrm{X}_{4}$ compartment as the most important criterion of the silicosis inducing action of the retained dust on the pulmonary parenchyma. As the retention of dust in the tracheobronchial lymph nodes is described in our model with a single compartment $\left(\mathrm{X}_{5}\right)$, its $\mathrm{AI}$ was used for estimating such action on lymph nodes.

To predict the degree of the antisilicotic effect of glutamate, we used the $A I /(A I)_{g}$ ratio, where $(A I)_{g}$ is the value of the AI computed for the model describing silica dust kinetics in rats drinking glutamate solution, and AI is the same value for rats drinking water. To compare this prediction with the experimental data ${ }^{15}$ we calculated $(A-B) /(C-D)$ ratios, where $C$ and $A$ are the indices of the state of the lungs (or lymph nodes) in rats exposed to silica dust with and without administration of glutamate respectively, $D$ and $B$ are the corresponding reference values for non-exposed rats.

It was found that the $\mathrm{AI} /(\mathrm{AI})_{\mathrm{g}}$ ratio for $\mathrm{X}_{4}$ by the end of a six month period of exposure was $2 \cdot 91$, and the experimental ratios $(A-B) /(C-D)$ for the wet weight of the lungs, total lipids, and total hydroxy- proline in the lung were $2 \cdot 14,2 \cdot 10$, and $2 \cdot 14$, respectively. The ratio $\mathrm{AI} /(\mathrm{AI})_{\mathrm{g}}$ for $\mathrm{X}_{5}$ was $1 \cdot 92$, and the $(A-B) /(C-D)$ ratios for the wet weight and the hydroxyproline content of lymph nodes were $2 \cdot 70$ and 1.93 respectively. For a model of this class (and keeping in mind that it was developed to imitate only the mechanism of dust retention, and does not include the mechanisms of its fibrogenic action as such) the accuracy of prediction is striking.

\section{Conclusion}

As was emphasised in the introduction, the basic aim pursued in the mathematical modelling of the process of dust retention in the pulmonary region of the lung and in tracheobronchial lymph nodes was to confirm the inherent consistency of the systems analysis as applied to this process based on our ideas on its mechanisms. Proceeding from these ideas we chose a structure for the multicompartmental model and imposed some limitations on its parameters. Then we showed the possibility of finding such sets of these parameters and such changes in part of them that ensured a satisfactory approximation to experimental data on the retention of quartz dust and basically correct simulation of such kinetic effects as the breakdown of the macrophagal clearance mechanism and its protection against damage. We think that this possibility is in itself of great importance.

The study provides evidence in favour of the concepts underlying the model, and thus the aim was achieved. The adequacy of the model was confirmed, which, in turn, will permit it to be used for predicting changes in the kinetics of dust retention in the lung and in lymph nodes under various conditions of exposure. This will enable definite changes in particular links of the phagocytic clearance mechanism to be predicted.

1 Vincent JH, Johnston AM, Jones AD, Bolton RE, Addison JA. Kinetics of deposition and clearance of inhaled mineral dusts during chronic exposure. Br J Ind Med 1985;42:707-15.

2 Smith TJ. Development and application of a model for estimating alveolar and interstitial dust levels. Ann Occup Hyg 1985;29:495-516.

3 Smith TJ. Exposure assessment for occupational epidemiology. Am J Ind Med 1987;12:249-68.

4 Bates TJ, Fish DR, Hatch Th F, et al. (Task Group on Lung Dynamics, ICRP.) Deposition and retention models for internal dosimetry of the human respiratory tract. Health Phys 1966;12:173-207.

5 Brain JD. Macrophages in respiratory tract. In: Fishman AP., Fisher AB, eds. Handbook of physiology section: respiratory system, Vol 1. Bethesda, Maryland: American Physiological Society, 1985:447-71.

6 Distefano JJ, Landaw EM. Multiexponential, multicompartmental, and noncompartmental modeling. 1. Methodological limitations and physiological interpretations. $A m$ J Physiol 1984;246:R651-64.

7 Katsnelson BA, Privalova LI. Recruitment of phagocytizing cells into the respiratory tract as a response to the cytotoxic action of deposited particles. Environ Health Perspect 1984, 55:313-25.

8 Privalova LI, Katsnelson BA, Yelnichnykh LN. Some peculiarities of the pulmonary phagocytotic response: dust retention kinetics and silicosis development during long term 
exposure of rats to high quartz dust levels. $\mathrm{Br} J$ Ind Med 1987;44:228-35.

9 Green GM. Alveolobronchial transport mechanisms. Arch Intern Med 1973;131:109-14.

10 Gross P, Smith KW. The topographic distribution of mineral dusts in some pneumoconiotic lungs. Dis Chest 1959;35: $140-54$

11 Heppleston AG. The disposal of dust in the lungs of silicotic rats. Am J Pathol 1962;40:492-506.

12 Katsnelson BA, Privalova LI, Kislitsina NS, Podgaiko GA. Correlation between cytotoxicity and fibrogenicity of silicosisinducing dusts. Med Lavoro 1984;75:456-62.

13 Stöber W, Einbrodt HJ, Klosterköter W. Quantitative studies of dust retention in animal and human lungs after chronic inhalation. In: Inhaled particles and vapours II. Oxford: Pergamon Press, 1967:40-17.

14 Tucker AD, Wyatt JH, Underly D. Clearance of inhaled particles from alveoli by normal interstitial drainage pathways. $J$ Appl Physiol 1973;35:719-32.

15 Morosova KI, Katsnelson BA, Rotenberg Yu S, Belobragina GV. A further experimental study of antisilicotic effect of glutamate. Br J Ind Med 1984;41:518-25.

16 Bowden DH, Adamson IYR. The pulmonary interstitial macro- phage as immediate precursor of the alveolar macrophage. $\mathrm{Am}$ $J$ Pathol 1972;68:521-8.

17 Privalova LI, Pashnina N Ya. Effect of macrophage breakdown products on the ${ }^{3} \mathrm{H}$-thymidine incorporation into pulmonary cells in rats. Arch Anat Histol Embriol 1989;46:66-9. (In Russian.)

18 Gross P, De Treville TP. The lung as an embattled domain against inanimate pollutants. A precis of mechanisms. Am Rev Respir Dis 1972;106:684-92.

19 La Belle Ch W, Brieger H. Patterns and mechanisms in the elimination of dusts from the lung. In: Inhaled particles and vapours. Oxford: Pergamon Press, 1961:356-68.

20 Baidosov VA, Katsnelson BA, Privalova LI. An approach to the mathematical analysis of the pattern organization of shortening the cumulative working period under exposure to harmful substances with marked cumulative ability, taking pulmonary dust retention as example. In: Mazurov ID, Smirnov AI, eds. Mathematical models of biological and medical systems. Sverdlovsk (USSR) Publishing House "Nauka", 1988:67-8. (In Russian.)

Accepted 24 June 1991.

\section{Vancouver style}

All manuscripts submitted to the $B r J$ Ind Med should conform to the uniform requirements for manuscripts submitted to biomedical journals (known as the Vancouver style).

The $\mathrm{Br} J$ Ind Med, together with many other international biomedical journals, has agreed to accept articles prepared in accordance with the Vancouver style. The style (described in full in $\mathrm{Br}$ Med J, 24 February 1979, p 532) is intended to standardise requirements for authors.

References should be numbered consecutively in the order in which they are first mentioned in the text by Arabic numerals above the line on each occasion the reference is cited (Manson' ${ }^{1}$ confirmed other reports ${ }^{2-5} \ldots$.). In future references to papers submitted to the $B r J$ Ind Med should include: the names of all authors if there are six or less or, if there are more, the first three followed by $e t$ al; the title of journal articles or book chapters; the titles of journals abbreviated according to the style of Index Medicus; and the first and final page numbers of the article or chapter.

Examples of common forms of references are:

1 International Steering Committee of Medical Editors. Uniform requirements for manuscripts submitted to biomedical journals. Br Med J 1979;1:532-5.

2 Soter NA, Wasserman SI, Austen KF. Cold urticaria: release into the circulation of histamine and eosino-phil chemotactic factor of anaphylaxis during cold challenge. N Engl J Med 1976;294:687-90.

3 Weinstein L, Swartz MN. Pathogenic properties of invading micro-organisms. In: Sodeman WA Jr, Sodeman WA eds. Pathologic physiology: mechanisms of disease. Philadelphia: W B Saunders, 1974:457-72. 